\title{
Entanglement dynamics of two independent Jaynes-Cummings atoms without rotating-wave approximation
}

\author{
Qing-Hu Chen ${ }^{1,2}$, Tao Liu ${ }^{3}$, Yuan Yang ${ }^{1}$, and Ke-Lin Wang ${ }^{4}$ \\ 1 Center for Statistical and Theoretical Condensed Matter Physics, \\ Zhejiang Normal University, Jinhua 321004, P. R. China \\ 2 Department of Physics, Zhejiang University, Hangzhou 310027, P. R. China \\ ${ }^{3}$ Department of Physics, Southwest University of Science \\ and Technology, Mianyang 621010, P. R. China \\ ${ }^{4}$ Department of Modern Physics, University of Science \\ and Technology of China, Hefei 230026, P. R. China
}

(Dated: November 10, 2018)

\begin{abstract}
Entanglement evolution of two independent Jaynes-Cummings atoms without rotating-wave approximation (RWA) is studied by an numerically exact approach. The previous results in the RWA are essentially modified in the strong coupling regime $(g \geq 0.1)$, which has been reached in the recent experiments on the flux qubit coupled to the LC resonator. For the initial Bell state with anti-correlated spins, the entanglement sudden death (ESD) is absent in the RWA, but does appear in the present numerical calculation without RWA. Aperiodic entanglement evolution in the strong coupling regime is observed. The strong atom-cavity coupling facilitates the ESD. The sign of detuning play a essential role in the entanglement evolution for strong coupling, which is irrelevant in the RWA. An analytical results based on an unitary transformation are also given, which could not modify the RWA picture essentially. It is suggested that the activation of the photons may be the origin of the ESD. The present theoretical results could be applied to artificial atoms realized in recent experiments.
\end{abstract}

PACS numbers: 


\section{INTRODUCTION}

Quantum entanglement, one of the most striking consequences of nonlocal quantum correlation, is fundamental in quantum physics both for understanding the nonlocality of quantum mechanics[1] and its role in quantum computations and communications[2]. The entanglement would undergo decoherence due to the unavoidable interaction with the environment. As a result, an initially entangled two-qubit system becomes totally disentangled after evolving for a finite time. This phenomena is called entanglement sudden death (ESD) [3] and has been recently demonstrated experimentally [4].

Many works are devoted to the ESD of the qubits coupled to an environment that results in irreversible loss, and the rotating-wave approximation (RWA) is made on the interaction of the qubits with the field $[3$, 5] . The effect of the counter-rotating terms on the ESD has been less studied, even for the Jaynes-Cummings (JC) model[6] where the qubit interacting only with the single-cavity mode. In fact, some investigations have also focused on the storing of entanglement in such a system[7]. So entanglement dynamics for two independent JC atoms is also of fundamental interest, and has been well studied only in the RWA[8-11].

On the other hand, the JC model is also closely related to condensed matter physics recently. It can be realized in some solid-state systems recently, such as one Josephson charge qubit coupling to an electromagnetic resonator [12], the superconducting quantum interference device coupled with a nanomechanical resonator [13, 14], and the most recently LC resonator magnetically coupled to a superconducting qubit[15]. In conventional quantum optics, the coupling between the two-level "natural" atom and the single bosonic mode is quite weak, RWA has been usually employed. With the progress of the fabrication, the artificial atoms may interact very strongly with on-chip resonant circuits[12 15], RWA can not describe well the strong coupling regime[16]. Therefore, it is highly desirable to explore the entanglement dynamics for two independent JC atoms without RWA.

However, due to the consideration of the counter-rotating terms, the photon number is not conserved, so the photonic Fock space has infinite dimensions, any solution without RWA is highly nontrivial. In the recent years, several non-RWA approaches[16 21] has been proposed in a few contexts. Especially, by using extended bosonic coherent states, three of the present authors and a collaborator have solved the Dicke model without RWA exactly in the numerical sense[17]. 
In this paper, we employ a numerically exact technique to solve JC model without RWA by means of extended bosonic coherent states. The correlations among bosons are added step by step until further corrections will not change the results. All eigenfunctions and eigenvalues can be obtained exactly. Based on the exact solutions to the single JC model, we can study the entanglement evolution of two JC atoms easily. Analytical results without RWA based on an unitary transformation are also presented. The paper is organized as follows. In Sec.II, the numerically exact solution to the JC model is proposed in detail and analytical results in terms of unitary transformation are also provided. The numerical results and discussions are given in Sec.III. The brief summary is presented finally in the last section.

\section{MODEL HAMILTONIAN}

The JC model can be written in the basis $(|\uparrow\rangle,|\downarrow\rangle)$ where the first index denoting spin-up and the second denoting spin-down

$$
H_{J C}=\frac{\Delta}{2} \sigma_{z}+\omega a^{+} a+\lambda\left(a+a^{+}\right) \sigma_{x}
$$

where $a^{+}$and $a$ are the bosonic annihilation and creation operators of the cavity, $\Delta$ and $\omega$ are the frequencies of the atom and cavity, $g$ is the atom-cavity coupling constant, and $\sigma_{k}(k=x, y, z)$ is the Pauli matrix of the two-level atoms. Here we set $\hbar=1$. The detuning is defined as $\delta=\omega-\Delta$.

In this paper, we describe two approaches to solve the single JC model without RWA. One is the numerically exact approach in terms of extended bosonic coherent states, the other is an analytical approach based on a unitary transformation.

Numerically exact approach.- In order to facilitate the calculation, we use a transformed Hamiltonian with a rotation around the $y$-axis by an angle $\pi / 4$, so $\sigma_{z} \rightarrow \sigma_{x}, \sigma_{x} \rightarrow-\sigma_{z}$. This rotation can also be described formally by the following transformation,

$$
H_{J C}^{\prime}=V H_{J C} V^{+}=-\frac{\Delta}{2} \sigma_{x}+\omega a^{+} a+\lambda\left(a+a^{+}\right) \sigma_{z}
$$

where

$$
V=\frac{1}{\sqrt{2}}\left(\begin{array}{ll}
1 & 1 \\
-1 & 1
\end{array}\right)
$$


By introducing the new operators

$$
A=a+g, B=a-g, g=\lambda / \omega
$$

we can observe that the linear term for the bosonic operator is removed, and only the number operators $A^{+} A$ and $B^{+} B$ are left. Therefore the wavefunction can be expanded in terms of these new operators as

$$
\left|\varphi^{\prime}(t)\right\rangle=\left(\begin{array}{c}
\sum_{n=0}^{N_{t r}} c_{1 n}|n\rangle_{A} \\
\sum_{n=0}^{N_{t r}} c_{2 n}|n\rangle_{B}
\end{array}\right)
$$

For $A$ operator, we have

$$
|n\rangle_{A}=\frac{A^{n}}{\sqrt{n !}}|0\rangle_{A}=\frac{(a+g / \omega)^{n}}{\sqrt{n !}}|0\rangle_{A} .
$$

The property of $B$ operator is the same.

The Hamiltonian (2) remains unchanged under the transformation level $1 \rightarrow$ level 2 and $a^{+}($or $a) \rightarrow-a^{+}($or $-a)$. So we can set $c_{2 n}= \pm(-1)^{n} c_{1 n}$ (or $\left.c_{1 n}= \pm(-1)^{n} c_{2 n}\right)$. The final more concise wavefunction is supposed as

$$
\left|\varphi^{\prime}(t)\right\rangle=\left(\begin{array}{l}
\sum_{n=0}^{N_{t r}} c_{n}|n\rangle_{A} \\
\pm \sum_{n=0}^{N_{t r}}(-1)^{n} c_{n}|n\rangle_{B}
\end{array}\right)
$$

The Schrö dinger equation is

$$
\omega\left(m-g^{2}\right) c_{m} \pm \frac{\Delta}{2} \sum_{n=0}^{N_{t r}} D_{m n} c_{n}=E^{( \pm)} c_{m}
$$

where

$$
D_{m n}=\exp \left(-2 g^{2}\right) \sum_{k=0}^{\min [m, n]}(-1)^{-k} \frac{\sqrt{m ! n !}(2 g)^{m+n-2 k}}{(m-k) !(n-k) ! k !} .
$$

The $l$-th eigenfunction $\left|\varphi^{\prime(l)}\right\rangle$ is then obtained, which can also be expressed in the original basis of Hamiltonian (1) as

$$
\left|\varphi^{(l)}\right\rangle=\left(\begin{array}{c}
\phi_{1}^{(l)} \\
\phi_{2}^{(l)}
\end{array}\right)=V^{+}\left|\varphi^{\prime(l)}\right\rangle
$$

The wavefunction at anytime then reads

$$
|\varphi(t)\rangle=\sum_{l=1}^{M_{0}} h^{(l)} \exp \left(-i E_{l} t\right)\left|\varphi^{(l)}(t)\right\rangle, M_{0}=2\left(N_{t r}+1\right)
$$


where $h^{(l)}$ is the coefficient to be determined.

For the two independent JC atoms, the eigenfunction of the total system is given by

$$
|\psi\rangle=\left|\varphi_{1}\right\rangle \otimes\left|\varphi_{2}\right\rangle
$$

where $\varphi_{i}$ is the eigenfunction the JC model $i(=1,2)$ with

$$
\left|\varphi_{i}\right\rangle=\left(\begin{array}{c}
\left|\phi_{i, 1}\right\rangle \\
\left|\phi_{i, 2}\right\rangle
\end{array}\right)
$$

The eigenvalue of the total system is $E=E_{1} \oplus E_{2}$. The $j-t h$ wavefunction for the total system can then be explicitly expressed in the basis $(|\uparrow \uparrow\rangle,|\uparrow \downarrow\rangle,|\downarrow \uparrow\rangle,|\downarrow \downarrow\rangle)$

$$
\left|\psi^{(j)}\right\rangle=\left(\begin{array}{c}
\phi_{1,1}^{(l)} \phi_{2,1}^{(k)} \\
\phi_{1,1}^{(l)} \phi_{2,2}^{(k)} \\
\phi_{1,2}^{(l)} \phi_{2,1}^{(k)} \\
\phi_{1,2}^{(l)} \phi_{2,2}^{(k)}
\end{array}\right), j=1,2,4\left(N_{t r}+1\right)
$$

The wavefunction of the total system at anytime then reads

$$
|\psi(t)\rangle=\sum_{j=1}^{2 M_{0}} f^{(j)} \exp \left(-i E_{j} t\right)\left|\psi^{(j)}\right\rangle, M_{0}=2\left(N_{t r}+1\right)
$$

where $f^{(j)}$ is the coefficient to be determined.

The initial two Bell states with anti-correlated and correlated spins, which are denoted by the Bell state 1 and 2 respectively for convenience, in the original base are the following if we use the column matrix

$$
\left|\psi_{\text {Bell }}^{(1)}\right\rangle=\left(\begin{array}{l}
0|00\rangle \\
\cos \alpha|00\rangle \\
\sin \alpha|00\rangle \\
0|00\rangle
\end{array}\right),\left|\psi_{\text {Bell }}^{(2)}\right\rangle=\left(\begin{array}{l}
\cos \alpha|00\rangle \\
0|00\rangle \\
0|00\rangle \\
\sin \alpha|00\rangle
\end{array}\right)
$$

where $|00\rangle$ denotes the photon number state in the two JC atoms, $f^{(l)}$ is determined by using $|\psi(0)\rangle=\left|\psi_{\text {Bell }}^{(i)}\right\rangle, i=1,2$.

For two-qubit states, entanglement can be quantified by the concurrence[22]. It can be calculated from the following reduced density matrix

$$
\rho=\operatorname{Tr}_{p h}(|\psi(t)\rangle\langle\psi(t)|)=\sum_{k, l=1}^{N_{0}} f^{(k)} f^{(l)} e^{-i\left(E_{k}-E_{l}\right) t} \Pi,
$$


where $4 \times 4$ matrix $\Pi$ is determined by $\left|\psi^{(j)}\right\rangle$. The 4 eigenvalues $\lambda_{i}$ of the matrix $\rho$ in decreasing order give the entanglement

$$
C^{A B}(t)=\max \left[0, \sqrt{\lambda_{1}}-\sqrt{\lambda_{2}}-\sqrt{\lambda_{3}}-\sqrt{\lambda_{4}}\right]
$$

Unitary transformation approach.- In order to treat JC model without RWA analytically, we perform a unitary transformation[18] on Hamiltonian (11) to eliminate the counterrotating wave term

$$
\begin{aligned}
H^{S} & =e^{S} H e^{-S}, \\
H & =H_{0}+H_{1}, H_{0}=\frac{\Delta}{2} \sigma_{z}+\omega a^{+} a, H_{1}=g\left(a^{+}+a\right)\left(\sigma_{+}+\sigma_{-}\right), \\
S & =\frac{g \xi}{\omega}\left(a^{+}-a\right)\left(\sigma_{+}+\sigma_{-}\right),
\end{aligned}
$$

where $\xi$ is a parameter to be determined. The transformed Hamiltonian can be expanded in terms of $g$ up to the $g^{2}$ term (higher terms are neglected), and we have

$$
\begin{gathered}
H^{S}=H_{0}+H_{1}^{S}+H_{2}^{S}+O\left(g^{3}\right), \\
H_{1}^{S}=H_{1}+\left[S, H_{0}\right]=g\left(\frac{\Delta}{\omega} \xi-\xi+1\right)\left(a^{+} \sigma_{-}+a \sigma_{+}\right)+g\left(-\frac{\Delta}{\omega} \xi-\xi+1\right)\left(a^{+} \sigma_{+}+a \sigma_{-}\right), \\
H_{2}^{S}=\left[S, H_{1}\right]+\frac{1}{2}\left[S,\left[S, H_{0}\right]\right]=-\frac{g^{2} \Delta}{(\omega+\Delta)^{2}} \sigma_{z}-\frac{g^{2}(\omega+2 \Delta)}{(\omega+\Delta)^{2}} .
\end{gathered}
$$

If we choose $\xi=\omega /(\Delta+\omega)$, the counter-rotating wave term can be eliminated, then we have the following renormalized JC Hamiltonian in a RWA form

$$
\begin{aligned}
H^{S} & =\frac{\Delta_{e f f}}{2} \sigma_{z}+\omega a^{+} a+g_{e f f}\left(a^{+} \sigma_{-}+a \sigma_{+}\right), \\
\Delta_{e f f} & =\Delta\left(1-\frac{2 g^{2}}{(\Delta+\omega)^{2}}\right), \\
g_{e f f} & =g\left(\frac{2 \Delta}{\omega+\Delta}\right),
\end{aligned}
$$

where a constant is removed. The effective detunning can be expressed in terms of original detunning $\delta$ as

$$
\delta_{e f f}=\omega-\Delta_{e f f}=\left(\delta+\frac{2 g^{2}(\omega-\delta)}{(2 \omega-\delta)^{2}}\right) .
$$

For $\delta=0$, we have

$$
\delta_{e f f}^{0}=\frac{g^{2}}{2 \omega}, \Delta_{e f f}^{0}=\Delta-\delta_{e f f}^{0}, \quad g_{e f f}^{0}=g
$$


Note that even for zero detunning, we have finite effective detunning after the unitary transformation. What is more, the non-RWA results within the unitary transformation approach is expected to be very close to the RWA ones in the weak coupling regime, because the effective $g$ is not changed, and only modification is the transition frequency which is reduced by a small amount proportional to $g^{2}$.

Follow the derivation for the RWA case in Ref. [8], if the initial state is the Bell state 1 , the concurrence for two identical JC atoms without RWA by the unitary transformation can be easily obtained

$$
C_{A B}(t)=|\sin 2 \alpha|\left[1-4 N^{2} \sin ^{2}(\nu t / 2)\right],
$$

where

$$
\begin{aligned}
N & =\frac{1}{2 \sqrt{1+\left[\frac{\left(\delta+\frac{2 g^{2}(\omega-\delta)}{(2 \omega-\delta)^{2}}\right)}{2\left(\frac{2 g}{1+1 /(1-\delta / \omega)}\right)}\right]^{2}}} \\
\nu & =\sqrt{\left(\delta+\frac{2 g^{2}(\omega-\delta)}{(2 \omega-\delta)^{2}}\right)^{2}+4\left(\frac{2 g}{1+1 /(1-\delta / \omega)}\right)^{2}} .
\end{aligned}
$$

For zero detunning, we have

$$
N_{0}=\frac{1}{2 \sqrt{1+\frac{g^{2}}{16 \omega^{2}}}}, \nu_{0}=\frac{g}{N_{0}} .
$$

This is to say, within unitary transformation approach, there is no ESD from the initial Bell state 1, similar to the RWA case.

Similarly, we can get the entanglement evolution from the initial Bell state 2

$$
C_{A B}(t)=\left[1-4 N^{2} \sin ^{2}(\nu t / 2)\right]\left(|\sin 2 \alpha|-8 N^{2} \sin ^{2}(\nu t / 2) \cos ^{2} \alpha\right),
$$

The ESD occurs for

$$
|\tan \alpha|<4 N^{2} \sin ^{2}(\nu t / 2) .
$$

Note form Eq. (28) that the value of $N_{0}$ is very close to the RWA one even for $g=1$, so it is expected that the non-RWA results within unitary transformation approach is nearly the same as that in RWA in a very large coupling regime. Moreover, the unitary transformation approach could not provide essentially different results, because final renormalized RWA Hamiltonian is derived for all calculations. In our numerically exact studies, the RWA-type Hamiltonian is not necessary, so some new results may be obtained. 

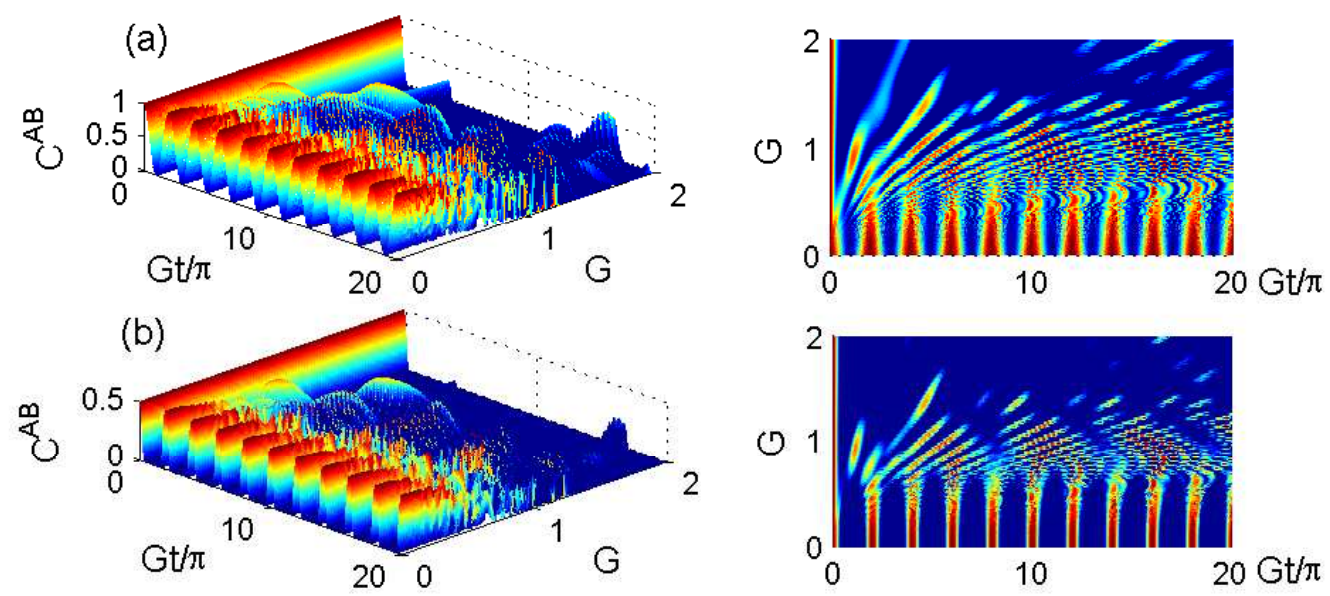

FIG. 1: (Color online) The concurrence for atom-atom entanglement with the initial atomic state: (a) Bell state 1 for $\alpha=\pi / 4$ and (b) Bell state 2 for $\alpha=\pi / 12$. $G=2 g$. The corresponding right figures are the bird's-eye view.

\section{RESULTS AND DISCUSSIONS}

We first study the evolution of the concurrence for atom-atom entanglement for two JC atoms without RWA when the states are initiated with the given Bell states ( $\alpha$ is fixed) ( (15). Actually, the dynamics of entanglement in bipartite quantum systems is sensitive to initial conditions[3, 23]

We consider the two same JC atoms for zero detuning. The concurrence for atom-atom entanglement as a function of time can be calculated with the use of Eqs. (13) and (14). As show in Fig. 1(a), for small $G=2 g$, the concurrence evolve as $\cos ^{2}(G t / 2)$, just follow that in RWA [8] for Bell state 1. For $G$ above 0.5, entanglement can drop to zero, which can not happen in the two JC atoms in RWA for Bell state 1. The entanglement can be revival irregularly. JC model is just $N=1$ Dicke model. We attribute this aperiodic entanglement evolution to the quantum chaos in finite Dicke model[24]. Without RWA, the system is integral, the emergency of the quantum chaos is impossible, so regular behavior is always observed [8, 10].

It is interesting that the area of the ESD become larger with increasing $G$. At very large coupling constant, the revival of entanglement will not happen, in sharp contrast with those observed in the RWA. Without RWA, the rule of the transfer of entanglement between the two-qubit subsystems derived in [9 11] may not hold due to presence of the counter-rotating 


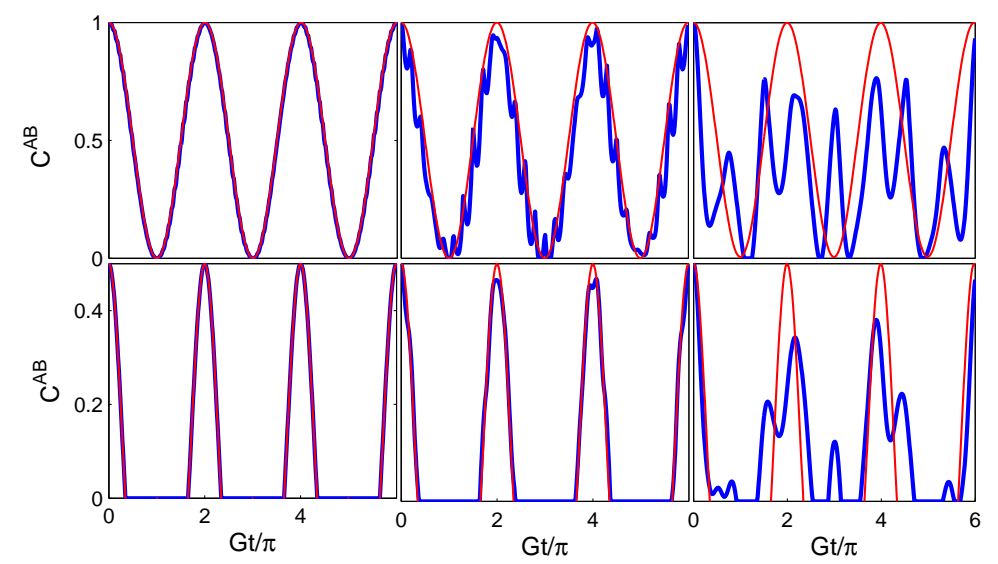

FIG. 2: (Color online) The concurrence for atom-atom entanglement with the initial atomic Bell state 1 (upper panel) and the Bell state 2 (down panel). From left to right column, $g=0.05,0.1$, and 0.3. The numerically exact results and those by the unitary transformation are denoted by the blue and red curves.
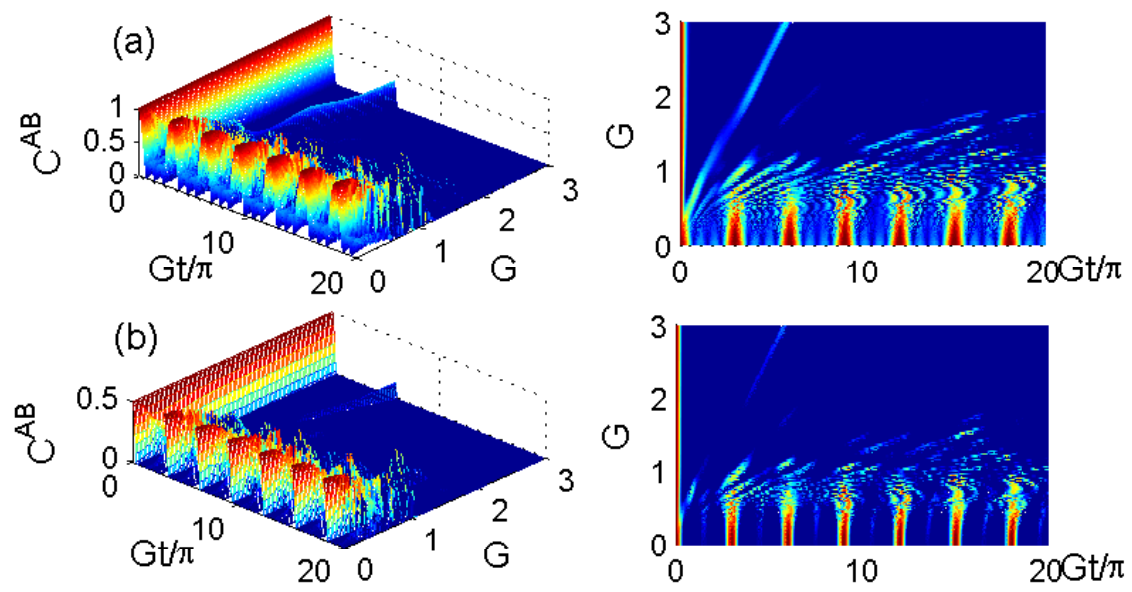

FIG. 3: (Color online) The concurrence for atom-atom entanglement with the initial atomic state: (a) Bell state 1 for $\alpha=\pi / 4$ and (b) Bell state 2 for $\alpha=\pi / 12 . \quad g_{1}=2 g_{2}, G=g_{1}+g_{2}$. The corresponding right figures are the bird's-eye view.

terms. Without RWA, we argue that there is no entanglement invariant because the photon number is not conserved.

For Bell state 2, the ESD can happen even in the RWA[8]. Without RWA, for small $G$, the entanglement dynamics show the same behavior. As shown in Fig. 1(b), the area of the ESD become larger with increasing $G$, and more wider than that in Bell state 1.

As we know, for weak coupling between the atom and the single bosonic mode, the RWA 
is a good approximation, the non-RWA treatment is not necessary. Our results for the entanglement dynamics also provide a evidence for this point. However, for strong coupling case, the entanglement dynamics demonstrate that the non-RWA should be considered essentially. As the new progress in the fabrication, some artificial atoms are just strongly coupled with the bosonic field.

The non-RWA JC model can be also treated by the unitary transformation approach. Is this approach really good enough in the strong coupling regime? We now present the analytical results based on this approach for zero detunning according to Eqs. (25) and (29), which are shown in Fig. 2. The corresponding numerically exact ones are also exhibited for comparison. It is obvious that the analytical results essentially deviate from the exact ones at $g=0.1$. Recent experiments on the LC resonator coupled to a flux qubit demonstrated that the system operates in the ultra-strong coupling regime $g=0.1$ [15], which crosses the limit of validity for RWA in the JC model. Since the analysis based on a unitary transformation could not essentially change the RWA results, the present numerically exact results are more necessary.

We next consider the two JC atoms with different atom-cavity coupling for zero detuning. Without loss of generality, we choose $g_{1}=2 g_{2}$. The concurrence for atom-atom entanglement as a function of time can also be calculated with the use of Eqs. (13) and (14). As shown in Fig. 3(a), for small $G$, the concurrence evolve as $\cos ^{2}(G t / 2)$, following that in RWA [8] for Bell state 1. As $G$ increases, entanglement can drop to zero and is almost not recovered, quite different from that in the two same JC atoms. We argue that two JC atoms with different coupling strength suppress the atom-atom entanglement. This point is confirmed by the evolution of the initial Bell state 2 as indicated in Fig. 3(b). No matter whether RWA is taken into account or not, the area of the ESD in the evolution of the initial Bell state 2 is larger than that in initial Bell state 1.

For more detail, we study the effect of the coupling strength on evolution of the concurrence for atom-atom entanglement. Without loss of generality, we only consider the two same JC atoms. As exhibited in Figs. 4 and 5, for weak coupling $g_{1}<10^{-3}$, no ESD is observed. For $g_{1}>10^{-3}$, the ESD appear, and its area is enlarged with $g$. At the same time, the periodicity of the entanglement evolution is destroyed.

One natural question is what the mechanism is for the ESD in two remote JC models. The photons should influence the atomic state considerably. We then calculate the average 


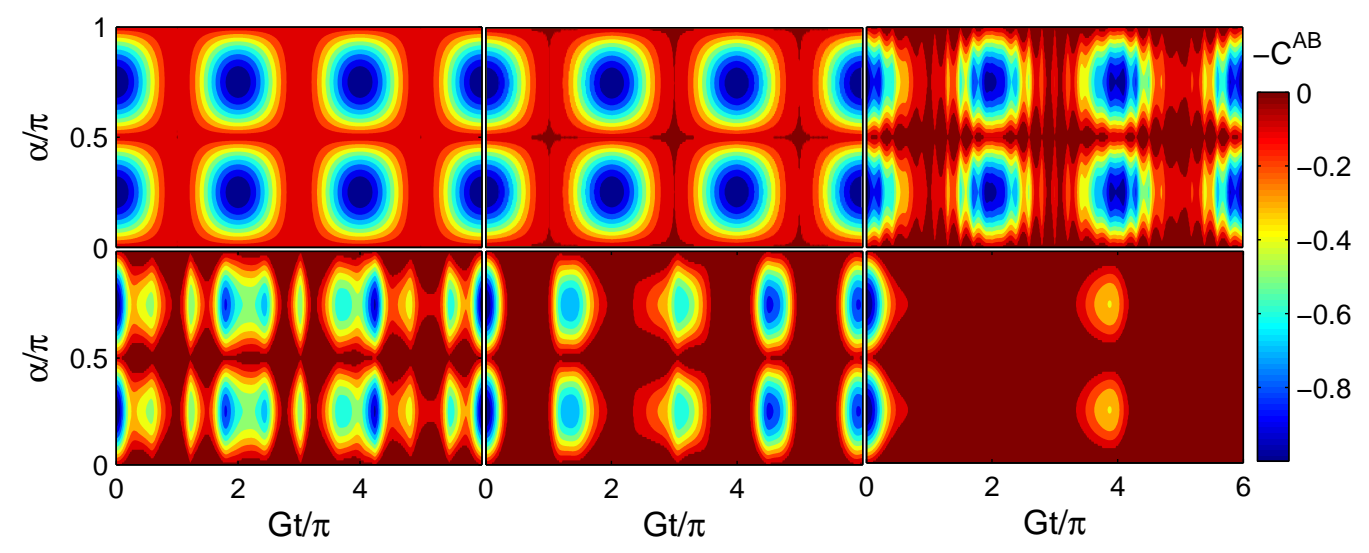

FIG. 4: (Color online) Histogram of the concurrence for atom-atom entanglement with the initial Bell state 1 . From left to right, $g=10^{-4}, 10^{-2}, 10^{-1}$ (top panel) and $g=0.25,0.5,1$ (bottom panel). $G=2 g$.

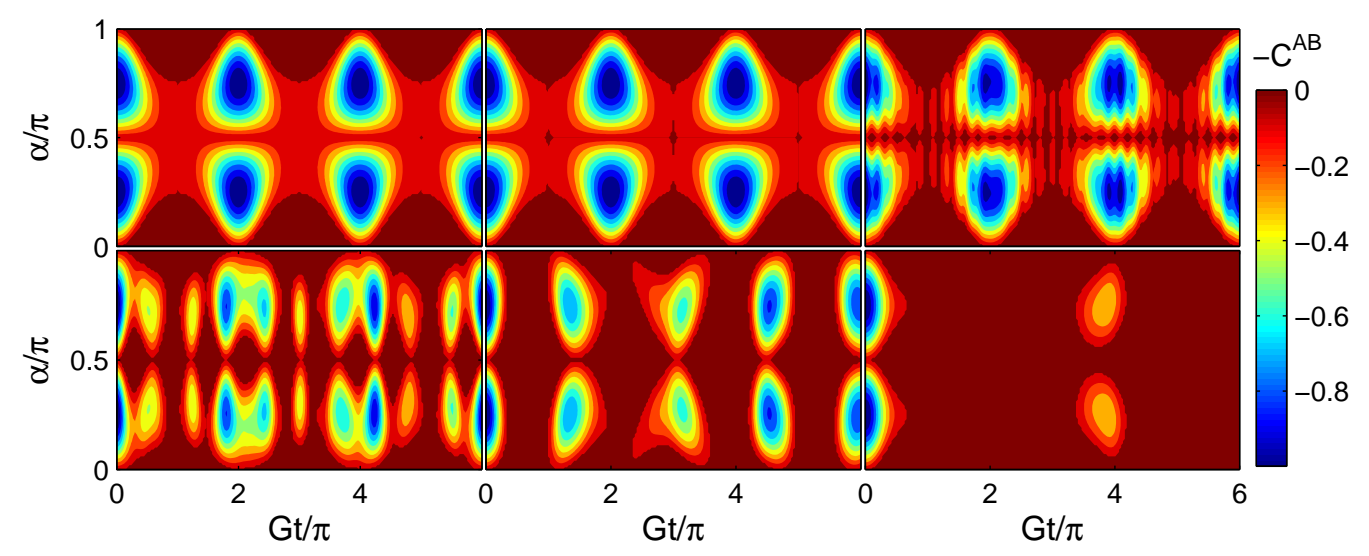

FIG. 5: (Color online) Histogram of the concurrence for atom-atom entanglement with the initial Bell state 2. From left to right, $g=10^{-4}, 10^{-2}, 10^{-1}$ (top panel) and $g=0.25,0.5,1$ (bottom panel). $G=2 g$.

photon number during the evolution. To show its role in the entanglement dynamics, we plot the average photon number together with entanglement for two limit coupling cases $g=0.001$ and 1 in Fig. 6 with initial Bell state 1 and Fig. 7 with initial Bell state 2. It is very interesting that the curves for the entanglement and the average photon number always show opposite behavior. It is highly suggested that the average photon number suppress the entanglement between two atoms considerably and sensitively for both weak and strong coupling. 

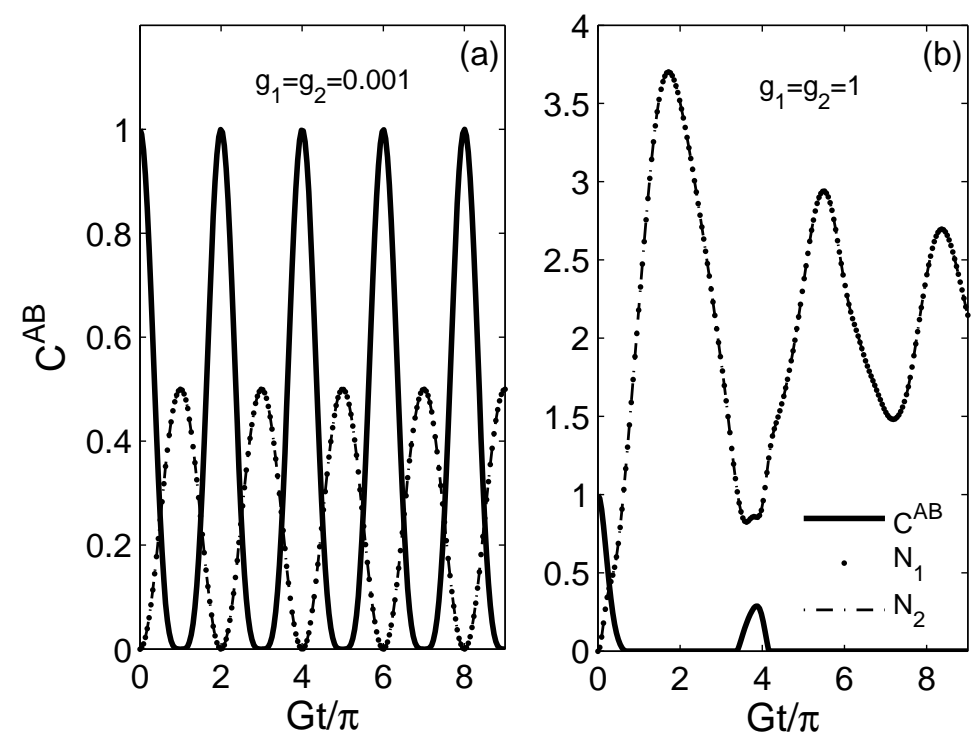

FIG. 6: The concurrence for atom-atom entanglement and the averaged photonic numbers for two identical JC atoms with the initial Bell state 1 for (a) $g=0.01$ and (b) $\mathrm{g}=1$. $G=2 g, \alpha=\pi / 4$.
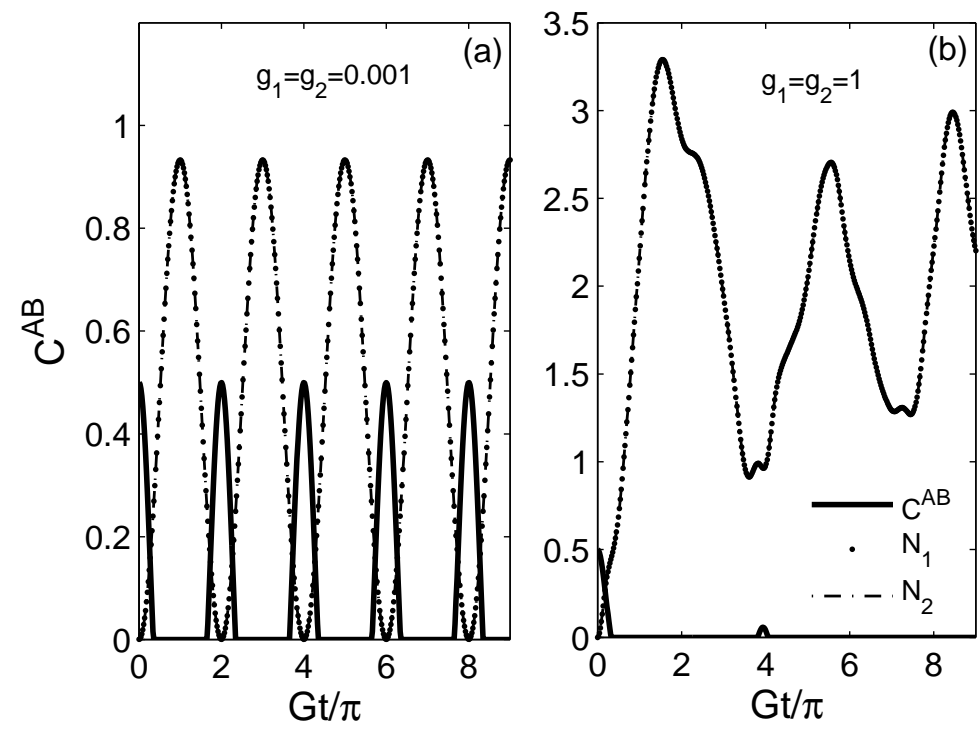

FIG. 7: The concurrence for atom-atom entanglement and the averaged photonic numbers for two identical JC atoms with the initial Bell state 2 for (a) $g=0.01$ and (b) $\mathrm{g}=1$. $G=2 g, \alpha=\pi / 12$. 
Finally, we turn to the effect of detuning on the entanglement evolution. It is found in

Ref. [10] that the atom-atom entanglement in RWA usually increases with ratio between detuning and coupling constant $|\delta| / g$ and is irrelevant with the sign of detuning. In other words, the entanglement decreases with $g$ for given detuning. Without RWA, it is not that case. After the unitary transformation, we have the renormalized JC Hamiltonian Eq. (20) in a RWA type. So entanglement evolution depends on $\left|\delta_{e f f}\right|$, which is related to both the magnitude and the sign of original detuning $|\delta|$ shown in Eq. (21). By Eqs. (22) and (23), the effective coupling constant can also be expressed as $g_{\text {eff }}=2 g /[1+1 /(1-\delta / \omega)]$ if the counter rotating-wave terms are taken into account after the unitary transformation. It follows that $g_{\text {eff }}$ decreases with $|\delta|$ for positive detuning and increases with $|\delta|$ for negative detuning. So in the strong coupling regime where the counter rotating-wave terms is required, the effect of detuning is very sensitive to the sign of detuning. The above discussions should be also suited qualitatively to the numerical exact results. We calculate the entanglement evolution of the initial Bell states 1 and 2 as a function of detuning. The results for different coupling constants ranging from weak to strong coupling regime are list in Fig. 8. The entanglement evolution is symmetric with detuning for the weak coupling and becomes asymmetric with the increase of the coupling constant. In the weak coupling regime, as shown in the left column, the entanglement increase with the value of detuning, and independent of the sign, similar to those in RWA. With the increase of the coupling, it is however observed that the entanglement decreases with the magnitude of the positive detuning, and increases with the magnitude of the minus detuning, in contrast to the case of RWA. In the strong coupling regime, the positive detuning stabilizes the entanglement and facilitates its revival, whereas the negative detuning suppresses the entanglement, facilitates its death, and reduces the period of entanglement revival.

\section{CONCLUSIONS}

In this paper, based on the exact solution for the single JC atoms, we are able to calculate exactly the entanglement evolution of the two independent JC atoms without RWA. The results are essentially different from RWA cases in the strong coupling regime. The analytical results based on an unitary transformation are also given. It can modify the RWA results and could not provide an essentially different ones. Initiated from the Bell state 1, the RWA 


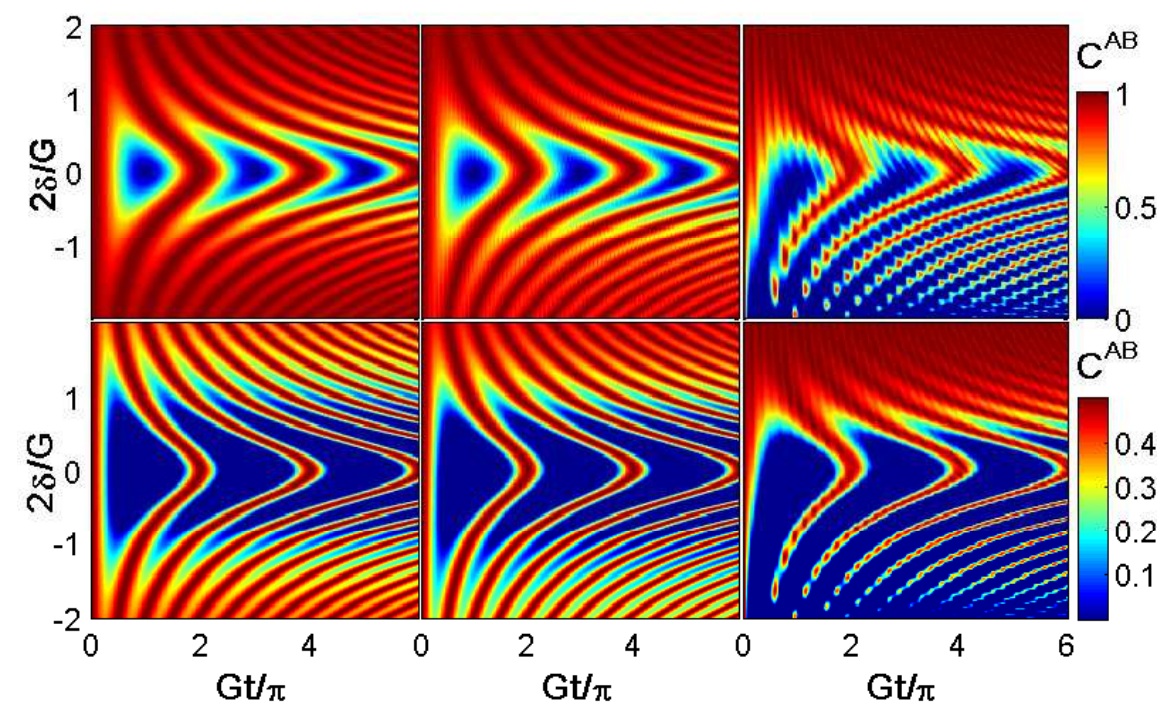

FIG. 8: Effect of detuning on the entanglement evolution of the Bell initial state 1 with $\alpha=$ $\pi / 4$ (top panel) and Bell initial state 2 with $\alpha=\pi / 12$ (bottom panel). From left to right column, $g=10^{-4}, 0.02,0.1 . \delta=\omega-\Delta, G=2 g$.

results show no ESD. The present numerically exact calculations for the non-RWA model show that the ESD could not be avoided, and the periodicity of entanglement evolution is destroyed by the presence of additional photons. We also suggest that the photons may suppress the entanglement and is just the origin of the ESD. The effect of the detunning on the entanglement evolution is also investigated. It is observed that the sign of detunning play a essential role in the strong coupling regime. The present theoretical prediction would be tested in a experimental study of ESD where the artificial atoms are made of circuit QED 12 15] if operating in the ultra-strong coupling regime. 


\section{ACKNOWLEDGEMENTS}

This work was supported by National Natural Science Foundation of China and National Basic Research Program of China (Grant Nos. 2011CB605903 and 2009CB929104).

[1] A. Einstein, B. Podolsky, and N. Rosen, Phys. Rev. 47, 777 (1935); J. S. Bell, Physics 1, 195 (1964).

[2] M. A. Nielsen and I. L. Chuang, Quantum Computation and Quantum Information (Cambridge University Press, Cambridge, England, 2000); C. H. Bennett and D. P. DiVincenzo, Nature (London) 404, 247 (2000).

[3] T. Yu and J. H. Eberly, Phys. Rev. Lett. 93, 140404 (2004); T. Yu and J. H. Eberly, Phys. Rev. Lett. 97, 140403 (2006).

[4] M. P. Almeida, F. de Melo, M. Hor-Meyll, A. Salles, S. P. Walborn, P. H. Souto Ribeiro, and L. Davidovich, Science 316, 579 (2007); J. S.Xu, C. F. Li, M. Gong, X. B. Zou, C. H. Shi, G. Chen, and G. C . Guo, Phys. Rev. Lett. 104, 100502 (2010).

[5] B. Bellomo, R. Lo Franco, and G. Compagno, Phys. Rev. Lett. 99, 160502 (2007); Q. J. Tong, J. H. An, H. G. Luo, and C. H. Oh, Phys. Rev. A 81, 052330 (2010).

[6] Jaynes E. T. and Cummings F. W., Proc. IEEE, 51, 89 (1963).

[7] J. Ye, D. V. Vernooy, and H. J. Kimbl, Phys. Rev. Lett. 83, 4987(1999).

[8] M. Yönac, T. Yu and J. H. Eberly, J. Phys. B: At. Mol. Opt. Phys. 39, S621(2006).

[9] M. Yönac, T. Yu and J. H. Eberly, J. Phys. B: At. Mol. Opt. Phys. 40, S45(2007)

[10] S. Chan, M. D. Reid, and Z. Ficek, J. Phys. B: At. Mol. Opt. Phys. 42, 065507(2009).

[11] I. Sainz and G. Bjork, Phys. Rev. A 76, 042313(2007)

[12] A. Wallraff et al., Nature (London) 431, 162 (2004);R. W. Simmonds et al., Phys. Rev. Lett. 93, 077003(2005).

[13] Y. Yu et al., Science 296, 889 (2002); I. Chiorescu et al., Science 299, 1869 (2003).

[14] I. Chiorescu et al., Nature 431, 159 (2004). J. Johansson et al., Phys. Rev. Lett. 96, 127006 (2006).

[15] P. Forn-Díaz, J. Lisenfeld, D. Marcos, J. J. García-Ripoll, E. Solano, C. J. P. M. Harmans, and J. E. Mooij, arXiv:1005.1559. 
[16] T. Liu, K. L. Wang, and M. Feng, EPL 86, 54003(2009).

[17] Q. H. Chen, Y. Y. Zhang, T. Liu, and K. L. Wang, Phys. Rev. A 78, 051801(R) (2008).

[18] H. Zheng, S.Y. Zhu, and M. S. Zubairy, Phys. Rev. Lett. 101, 200404 (2008).

[19] T. Liu, Y. Y. Zhang, Q. H. Chen, and K. L. Wang, Phys. Rev. A 80, 023810(2009).

[20] L. Amico et al., Nucl. Phys. B787, 283(2007).

[21] Y. Y. Zhang, Q. H. Chen, and K. L. Wang, Phys. Rev. B 81, 121105 (R)(2010)

[22] W. K. Wootters, Phys. Rev. Lett. 80, 2245 (1998).

[23] K. Roszak and P. Machnikowski, Phys. Rev. A 73, 022313 (2006).

[24] C. Emary and T. Brandes, Phys. Rev. Lett. 90, 044101(2003); Phys. Rev. E 67, 066203(2003). 